\title{
Isosurface-Based Generation of Hulls Encompassing Neuronal Pathways
}

\author{
Dorit Merhof $^{a}$ b Martin Meister $^{\mathrm{a}}$ Ezgi Bingöla ${ }^{\mathrm{a}}$ Christopher Nimsky ${ }^{\mathrm{b}}$ \\ Günther Greiner ${ }^{\mathrm{a}}$ \\ ${ }^{a}$ Computer Graphics Group, and b Department of Neurosurgery, University of Erlangen-Nuremberg, \\ Erlangen, Germany
}

\section{Key Words}

Neuronavigation • Diffusion tensor imaging $\cdot$ Fiber tracking $\cdot$

White matter tracts $\cdot$ Hulls ferent tract systems, which can be used as a basis for intraoperative visualization in the OR microscope. Distance measurements further confirm the accuracy of the hulls.

Copyright $\odot 2009$ S. Karger AG, Basel

\section{Introduction}

Diffusion tensor imaging (DTI) $[1,2]$ is a magnetic resonance imaging (MRI) technique which has gained importance in recent years. It reflects the organization and structure of fibrous tissue such as white matter in vivo which is of high value for neurosurgery and other disciplines. DTI measures the diffusion of water molecules which is anisotropic in fibrous tissue such as white matter. The visualization of DTI data is nontrivial due to the complex tensor information that has to be processed, resulting in a variety of different approaches. In principle, three major strategies can be distinguished: direct visualization of the tensor information per voxel is achieved by glyph-based techniques. This approach conveys the tensor information using glyph representations such as ellipsoids [3] or superquadric tensor glyphs [4]. As a result, the tensor information is comprehensively visualized. However, this representation is difficult to interpret and is rarely used for medical diagnosis and planning.

\section{KARGER}

Fax +4161306 1234 E-Mail karger@karger.ch www.karger.com

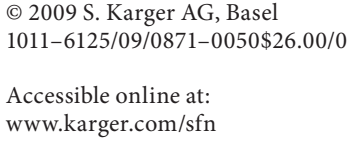

Dr.-Ing. Dorit Merhof

Computer Graphics Group, University of Erlangen

Am Weichselgarten 9, DE-91058 Erlangen (Germany)

Tel. +49 9131852 9919, Fax +499131 8529931

E-Mail dorit.merhof@informatik.uni-erlangen.de 
Alternatively, diffusion metrics have been developed that characterize the complex tensor information using a scalar value only. An example for a scalar metric is the fractional anisotropy (FA) [1] which is a measure for anisotropic diffusion. In medical application, 2-dimensional slice images of scalar metrics such as FA [1] show the location of white matter structures and allow to inspect a tumor and surrounding white matter. However, directional information showing the course of the underlying white matter tracts is difficult to convey even if color coding according to the direction of main diffusion is applied.

From the medical point of view, fiber tracking $[5,6]$ approaches are the preferred technique for visualizing DTI data and are applied in pioneering clinics for planning and navigation in neurosurgery $[7,8]$. These algorithms take advantage of the major eigenvector of each tensor which correlates with the direction of main diffusion. Streamline techniques known from flow visualization are then applied to propagate streamlines. The resulting streamline bundles represent the shape and course of white matter tracts and provide a meaningful 3-dimensional visualization of major tract systems.

In neurosurgery, major white matter structures must be protected against injury in order to avoid postoperative neurological deficits. Especially in cases where the tumor is very close to a tract system, or difficult to access, the reconstructed bundles are of great importance. For this reason, essential tract systems such as the optical tract (vision), the pyramidal tract (motor tract system) or the corpus callosum (connection between the two hemispheres) are reconstructed prior to surgery for preoperative planning. In addition to standard MRI data, the streamline bundles reconstructed from DTI data provide valuable information about the location and extent of white matter structures.

However, for intraoperative visualization, streamline representations are not adequate. The neuronavigation system is capable of superimposing boundary curves of important structures on the operating room $(\mathrm{OR})$ microscope image in order to guide the surgeon during the intervention. This is shown in figure 1, where the borders of the tumor and the optical tract are superimposed on the microscope view in order to display the relation between tumor tissue and neuronal structures. The dashed lines indicate the maximum extent of the respective structures. During surgery, drawn-through boundary curves appear as soon as the OR focus plane intersects these structures. The boundary curves provide a clear representation and explicitly show the extent of the fiber

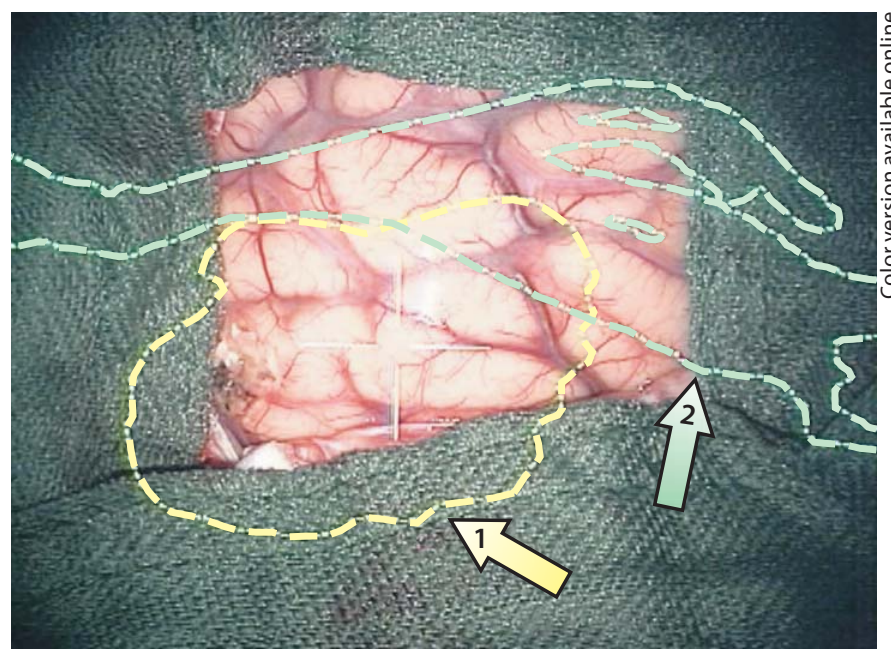

Fig. 1. View through the OR microscope. The border of the tumor (arrow 1; yellow in the online version) as well as functional structures such as the optical tract (arrow 2; green in the online version) are superimposed on the microscope image.

tract, whereas a direct visualization of fiber segments would result in a confusing, overloaded region of short line segments, with no clear boarder.

For this purpose, hulls encompassing the streamline bundles are necessary for intraoperative visualization. In this work, we present a novel hull approach based on rasterization and isosurfaces, combined with filtering techniques, for medical application. The advantages of the approach are its robustness and the possibility to control the tightness of wrapping. As a result, precise hulls are generated which are the basis for intraoperative visualization.

The paper is organized as follows: first, a survey of related work about fiber tracking and hull algorithms is given, followed by an introduction to DTI data acquisition and tensor reconstruction as well as fiber tracking. The next section is divided into four parts presenting the construction of hulls based on rasterization, volume filtering, isosurface extraction and surface filtering. The visualization of the resulting hulls is addressed subsequently. Finally, the results of our approach are presented and discussed.

\section{Related Work}

Streamline bundles resulting from fiber tracking indicate the location and orientation of the underlying white matter structures within the human brain. For surgical 
planning, they are the preferred DTI visualization technique. In addition to standard approaches based on streamline propagation $[5,6]$, a wide variety of different approaches is available due to extensive research in this field [9-12]. As a result, all these algorithms generate bundles of lines that approximate the shape and location of the underlying white matter tracts.

In principal, various approaches for generating surfaces based on segmented data have been presented in the literature. The marching cubes algorithm [13], which is maybe the most popular surface extraction approach, computes an isosurface for discretely sampled volume data. An extension of the marching cubes algorithm denoted as dual marching cubes [14] generates an isosurface composed of quad faces in order to circumvent thin triangles. Another strategy denoted as constrained elastic surface net [15] places vertices within the surface voxels of a segmented region. The mesh connecting the vertices is relaxed in order to smooth the surface, while constraining the vertices to remain within the respective surface voxel. Further surface extraction approaches are summarized and discussed in Nielson [14].

In order to wrap fiber bundles, two different techniques have been presented in recent research. The first approach [16] computes the centerline of the fiber bundle and defines ellipses encompassing the fibers in a series of planes perpendicular to the centerline. The ellipses of subsequent planes are then connected using a triangular mesh, resulting in a tube encompassing the fiber tract. This approach has been developed in conjunction with automatic clustering [17-19], which ensures that single streamlines within a cluster or bundle pursue a similar course. For this reason, the technique works well for elongated white matter tracts where the centerline is well defined. However, for tract systems such as the corpus callosum encompassing fibers with significantly varying direction, the approach will fail. Another problem occurs, if the fiber tract branches, which would require a splitting centerline or the definition of several ellipses per plane.

The second approach [20] considers the point cloud of the sampling points of all streamlines which serve as a basis for tetrahedralization. The outer faces of the resulting tetrahedral mesh describe the convex hull of the point cloud. In order to reveal the shape of the fiber tract, an $\alpha$-shaped algorithm is applied that removes outer tetrahedra above a certain edge length in an iterative procedure. This approach provides hulls that tightly fit the underlying fiber bundles since the sampling points of the fibers are reused. However, in case of sparse bundles, holes may occur if the threshold for tetrahedra removal is too low or if an adaptive step size has been applied during fiber tracking.

For this reason, an alternative technique for hull generation is presented in this work, overcoming the drawbacks of current approaches. This approach was developed to meet the requirements of surgery and produces user-defined hulls for clinical application. The processing steps for hull generation have been thoroughly investigated and are harmonized with each other in order to provide hulls of high precision. For this purpose, filtering and surface extraction algorithms well known from the literature are applied, which have gained a certain reputation in medical data processing. In a first processing step, the fibers are rasterized and a volume filter is applied to the rasterized volume. In a second step, a hull surface is extracted using the marching cubes algorithm. The resulting surface is smoothed using surface filtering approaches, where the number of iterations is used to control volume shrinkage and the tightness of the fitted hull. As a result, tight and smooth hulls are generated for arbitrary tract shapes, which are very well suited for intraoperative navigation during surgery.

\section{Data Acquisition and Preprocessing}

Starting with DTI acquisition, different processing steps are necessary prior to hull generation, which are outlined in what follows. This section explains the principle of DTI as well as subsequent data processing. Finally, the applied fiber tracking approach is described.

\section{Image Data}

DTI is an MRI acquisition technique which measures the diffusion of water molecules. In fibrous tissue, such as white matter, which consists of long, cylindric cells, anisotropic diffusion is observed. For this reason, the diffusion characteristics of water provide information about the underlying tissue structure. Based on six diffusion datasets acquired for different gradient directions and a reference dataset measured without gradient, a symmetric second-order tensor is computed per voxel which characterizes the properties of local diffusion. Eigenanalysis of the tensor yields the eigenvectors and the respective eigenvalues $\lambda_{i}(i=1,2,3)$ which characterize diffusion. Anisotropic diffusion, which is related to underlying white matter structures, occurs if the dominant eigenvalue $\lambda_{1}$ is much greater than both other eigenvalues. The associated dominant eigenvector indicates the direction of main diffusion. 


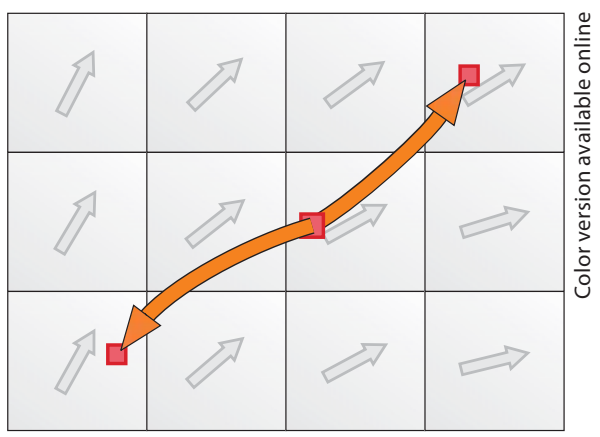

Fig. 2. Schematic outline of streamline propagation applied for fiber tracking. According to the underlying vector field, each streamline extends in forward and backward direction until a termination criterion is reached.

For this work, DTI data were acquired in patients with brain tumors, using a Siemens MR Magnetom Sonata Maestro Class 1.5-tesla scanner. The resulting datasets had a resolution of $128 \times 128 \times 60$ voxels and a voxel size of $1.875 \times 1.875 \times 1.9 \mathrm{~mm}^{3}$. Additionally, an anatomical MRI $\mathrm{Ml}_{T 1}$ sequence $(256 \times 256 \times 160$ voxels, $1 \times$ $1 \times 1 \mathrm{~mm}^{3}$ voxel size) was acquired.

\section{Fiber Tracking}

Fiber tracking approaches based on streamline propagation $[5,6]$ select seed points within the volume and propagate fibers until certain termination criteria are reached. In our implementation, seed points are positioned in regions with anisotropic diffusion and serve as starting points for fiber tracking. For each seed point, a streamline is propagated in both directions using streamline integration. For this purpose, a fourth-order Runge-Kutta integration scheme is applied which uses component-wise trilinear tensor interpolation and computation of the eigensystem in order to determine the direction of the main eigenvector at arbitrary positions. The maximum step size for each integration step is defined by the user and can either be constant or adaptive [21]. Propagation terminates, if the streamline leaves regions with anisotropic diffusion, which is expressed by a threshold based on FA [5]. Streamlines are also terminated, if they have reached a predefined maximum length. An outline of streamline propagation is given in figure 2 .

For the definition of separate tract systems which can be attributed to a specific function, tract selection approaches are required. In clinical application, regions of interest (ROIs) defined by a medical expert are typically used for this purpose [8]. In this work, one or more ROIs were segmented in axial slices that cross the respective tract, based on anatomical knowledge. Based on these ROIs, the fiber tracking algorithm generates streamlines that cross the ROIs. As a result, separate tract systems such as the pyramidal tract, the optical tract or the corpus callosum can be obtained.

\section{Isosurface-Based Generation of Hulls}

For the generation of hulls based on the reconstructed fiber tract bundles, a series of processing steps has to be accomplished, which are outlined in this section.

\section{Rasterization}

In order to encompass the fiber bundle with a wrapping surface, the volume occupied in 3-dimensional space is determined in a first step which is achieved with rasterization.

For this purpose, the Bresenham algorithm [22] is employed, which is the standard concept for rasterization, originally designed for lines in 2-dimensional discrete space. For rasterization in 3-dimensional space, it has been adapted to 3 dimensions [23]. The 3-dimensional version of the Bresenham algorithm projects the 3-dimensional line on 2-dimensional planes and executes the 2-dimensional Bresenham algorithm on each plane.

Since the fiber bundles consist of multiple streamlines which are composed of a series of line segments, the 3dimensional Bresenham algorithm is executed for each line segment by taking the start and endpoint of the line segment as input values. The rasterization result is a binary volume that approximates the fiber bundle in discrete 3 -dimensional space. With respect to the resolution of the grid, two contradicting criteria are encountered: the grid must be fine enough in order to maintain the shape of the tract after rasterization, but at the same time, the tract should be represented by connected rasterized voxels without holes. A good trade-off between these criteria is provided if the grid size is above the sampling distance between single fibers.

For a maximum fiber tracking step size below the rasterization resolution, it would be just as well possible to mark voxels containing a tract vertex. However, rasterization based on the 3-dimensional Bresenham algorithm ensures that streamlines with arbitrary sampling features, such as an adaptive step size or a step size above the grid resolution, are correctly processed. 

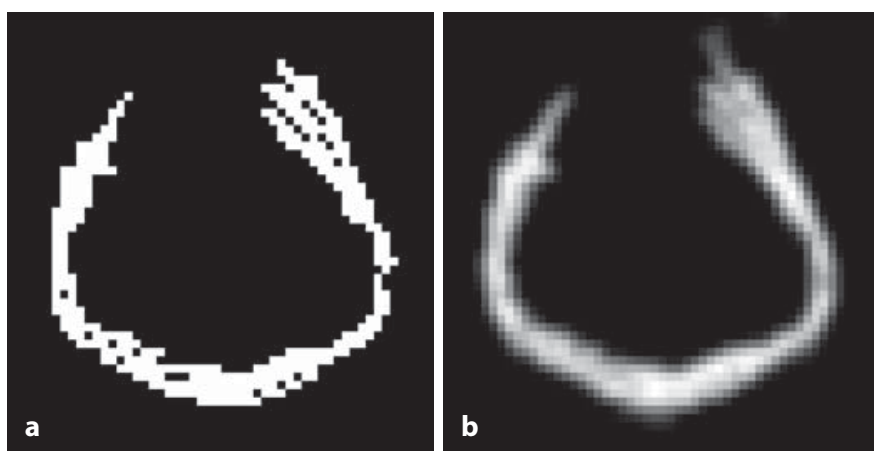

Fig. 3. Comparison of volume after rasterization using 3-dimensional Bresenham (a) and additional gaussian volume filtering (b), shown for a coronal slice of the corpus callosum.

\section{Volume Filtering}

The binary volume generated with the 3-dimensional Bresenham algorithm needs further preprocessing, in order to serve as input for a surface extraction algorithm, since its discrete nature would result in jaggies. In order to reduce these aliasing artifacts, filter operations are commonly applied on the volume. For this purpose, a 3 dimensional gaussian filter is used. This filter kernel suppresses high frequencies in the spatial domain and smoothes the rasterized volume. In figure 3 , the effect of gaussian volume filtering is shown for a coronal slice of the corpus callosum.

Application of the 3-dimensional gaussian filter not only smoothes the volume, but also results in a slight volume expansion. However, this is tolerable if an appropriate value for isosurface extraction is used, or if surface filters are applied later on, which cause a slight volume shrinkage. In order to maintain the shape of the tract system during volume filtering, it is advisable to use a small gaussian kernel of the size of $3 \times 3 \times 3$. In addition to gaussian volume filtering, morphological operations could be applied in order to fill potential holes and to further smooth the volume. However, for the processed tract systems consisting of dense fiber bundles, application of the gaussian volume filter was sufficient.

\section{Surface Extraction}

Based on the rasterized and filtered volume, surface extraction is applied. For this purpose, the marching cubes algorithm [13] is employed. In order to extract an isosurface encompassing all fibers, a low isovalue has to be selected. The rasterized and Gauss-filtered volume comprises gray values between 0 and 255; for isosurface extraction an isovalue between 0 and 10 is advisable. In

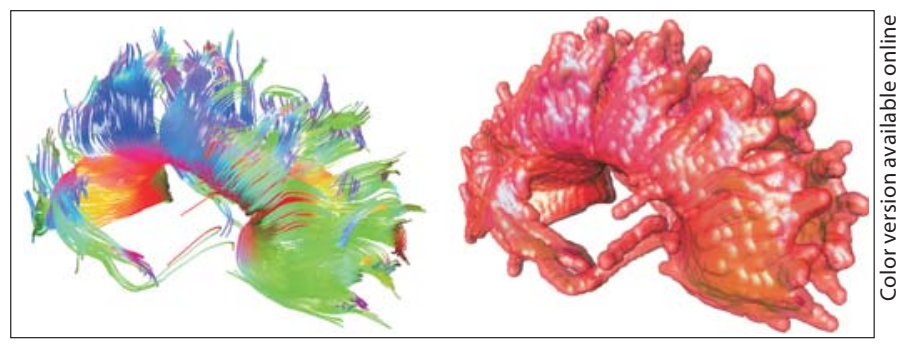

Fig. 4. Initial isosurface extracted with marching cubes for an isovalue of 1 .

figure 4, an isosurface extracted by marching cubes based on a rasterized and Gauss-filtered volume is shown. Even though the marching cubes algorithm works on a filtered volume, the extracted surface features terracing artifacts. For this reason, surface filtering is applied in the next step in order to attenuate these artifacts resulting in a smooth hull which better fits the underlying fiber structures.

\section{Surface Filtering}

In spite of volume filtering, the hulls resulting from isosurface extraction suffer from unevenness and consequently lack tightness. For this reason, three surface filters are presented in this section, which are based on the umbrella operator [24]. The filter parameters are adjusted by the user in order to generate tight and smooth hulls.

\section{Laplace Filter}

A discrete Laplace filter based on angle weights was originally proposed by Pinkall and Polthier [25]. In the meantime, the Laplace filter has become a standard approach for mesh smoothing [24, 26-28]. Based on the topological neighborhood, each vertex is repositioned and the mesh gets smoother within a few iterations. In each iteration, the Laplace filter traverses all surface vertices and moves each vertex $\vec{p}$ to the geometric center of the $n$ topological neighbor vertices $\vec{q}_{i}$. Since this approach leads to a considerable shrinkage of the surface, a weighting factor $\lambda$ is commonly applied. This factor has the effect that $\vec{p}$ is not completely moved to the center of its topological neighbors, but only for a portion $\lambda$ of the movement vector. Accordingly, the smoothed position $\vec{p}^{\prime}$ of vertex $\vec{p}$ is computed as follows:

$$
\vec{p}^{\prime}=\vec{p}+\lambda \frac{1}{n} \sum_{i=0}^{n-1}\left(\vec{q}_{i}-\vec{p}\right)
$$



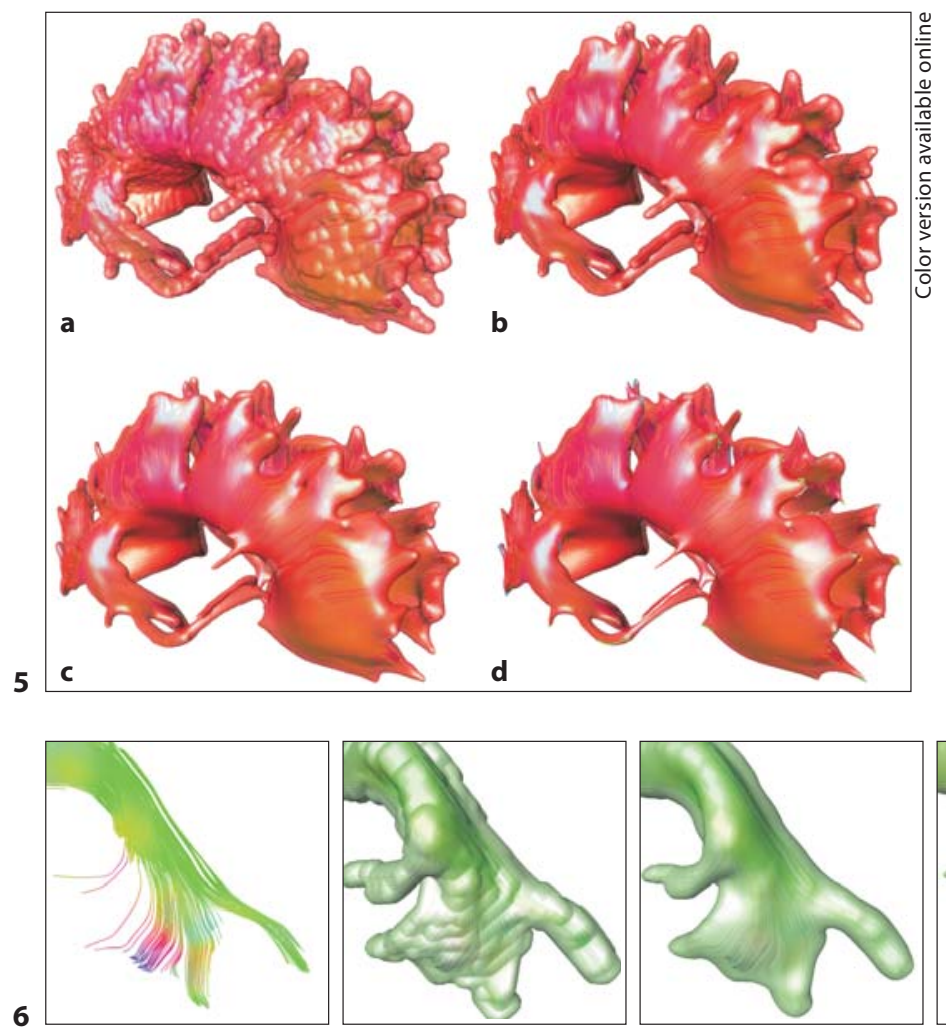

Fig. 5. Surface filtering with a scale-dependent Laplace filter after 0 (a), 25 (b), 50 (c) and 75 (d) iterations and a filter value $\lambda=0.5$. With increasing iteration number, the hull gets tighter.

Fig. 6. Zoomed view of the optical tract. Fibers and hull surface after $0,10,25,50$ and 75 surface filter iterations (scale-dependent Laplace filter) for a filter value $\lambda=0.5$ (from left to right).

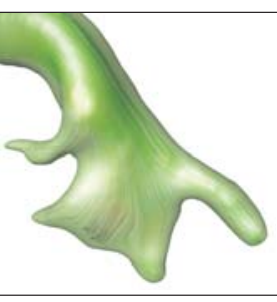

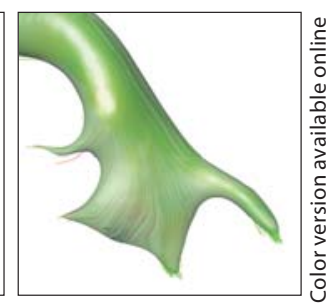

Scale-Dependent Laplace Filter

The Laplace filter based on the umbrella operator does not take into account the angles between triangles or the edge length, which leads to the same smoothing result for different neighborhood configurations. However, the length of edges in the neighborhood provides more information about the configuration of a triangulation which is important for good smoothing results. For this reason, the scale-dependent Laplace filter [27] scales the umbrella operator with the sum $E$ of the lengths of all neighbor edges. The resulting filter computes the smoothed position $\vec{p}^{\prime}$ of a vertex from its old position $\vec{p}$ and its neighbors $\vec{q}_{i}$ as described previously, but the vectors to the neighbor vertices are normalized and the sum of edge lengths $E$ is used in order to scale the resulting movement vector:

$$
\vec{p}^{\prime}=\vec{p}+\frac{\lambda}{E} \sum_{i=0}^{n-1} \frac{\left(\vec{q}_{i}-\vec{p}\right)}{\left\|\vec{q}_{i}-\vec{p}\right\|} \text {, with } E=\sum_{i=0}^{n-1}\left\|\vec{q}_{i}-\vec{p}\right\|
$$

In figures 5 and 6 , smoothing results obtained with the scale-dependent Laplace filter are shown. Depending on the number of iterations, a slight but constant volume shrinkage is observed. At the same time, the surface gets smoother and terracing artifacts disappear. The filter pa- rameter $\lambda$ as well as the number of iterations is controlled by the user in order to obtain a hull surface tightly fitting the underlying fiber tract. In addition to the isovalue, the filter parameters thus allow subtle fitting of the hull in order to obtain the desired surface quality.

\section{Visualization}

Lighting plays an important role for investigating the structure and the quality of the hull surfaces. For this reason, shading is essential for a meaningful visualization. For this purpose, per-pixel shading is used which provides high visualization quality. This is accomplished with shader programs which are supported by current graphics hardware.

Transparency is applied for quality control and provides excellent support for comparing the generated surfaces with the underlying streamline bundles. Semitransparent rendering of a single hull is straight forward. In case of several surfaces generated with different surface generation parameters enclosing the same tract, correct semi-transparent rendering is achieved by performing front-face culling in a first pass and back-face culling 
Fig. 7. Fiber tracts for the optic (green in the online version) and pyramidal (blue in the online version) tract (a) and the corresponding hull surfaces generated with an isovalue of 5 and a scaledependent Laplace filter with a filter value of 0.5 and 40 iterations (b).

Fig. 8. Pyramidal tract of different brain tumor patients undergoing surgery. Based on the fiber tracking results, hulls were generated (isovalue 5, scale-dependent Laplace filter with $\lambda=0.5,50$ iterations) (a-d). Semi-transparent visualization of the hull in combination with the fiber tracking results $(\mathbf{e}-\mathbf{h})$ facilitates the adjustment of the surface extraction parameters in order to obtain tight hulls.
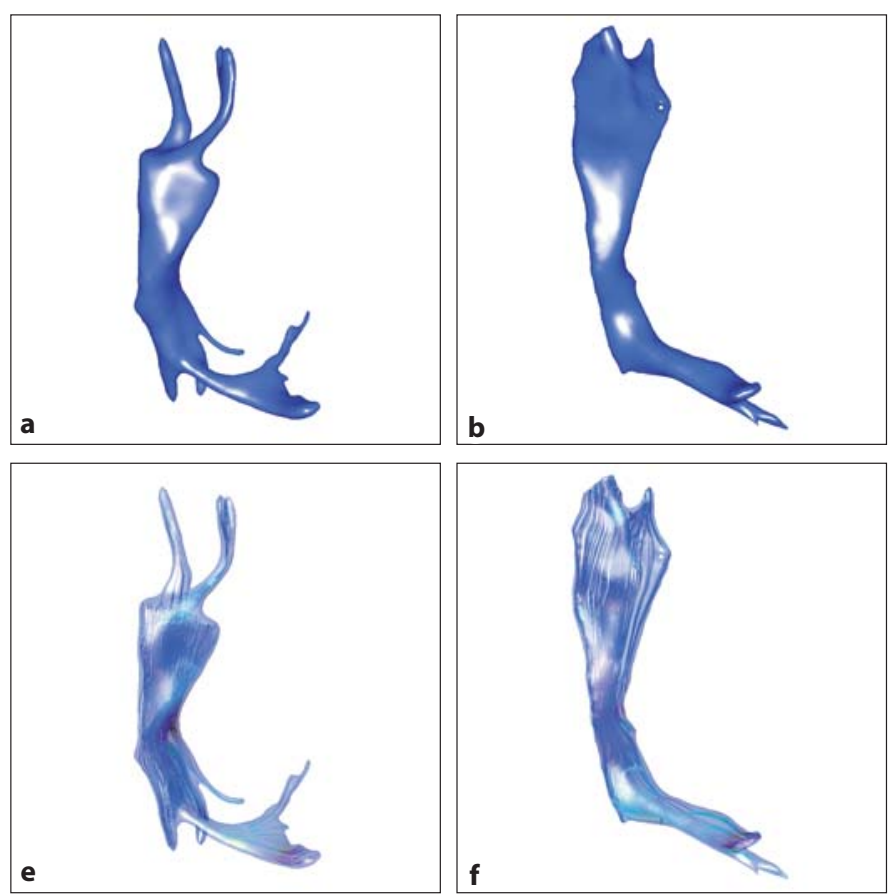
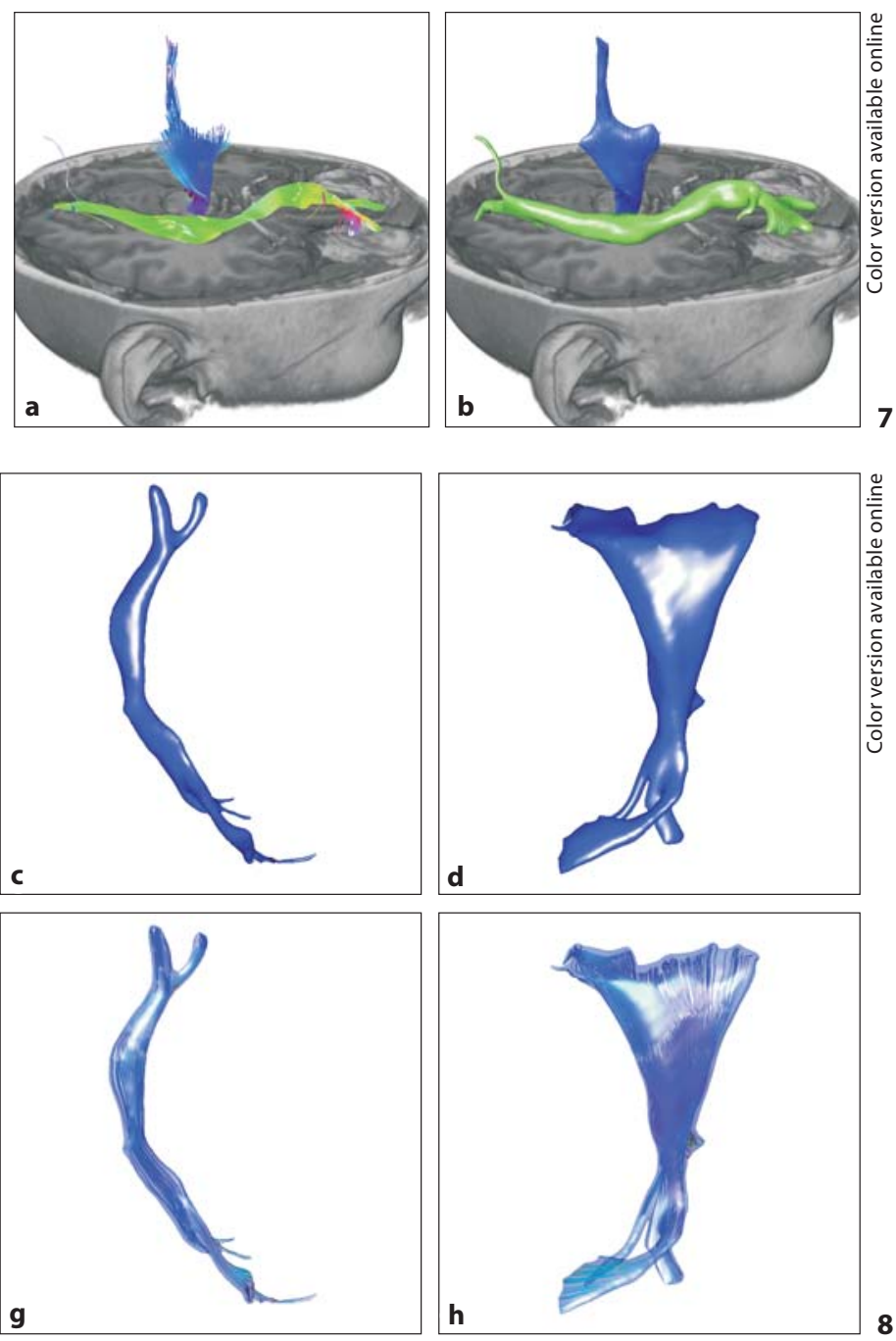

in a second pass. In the first pass, the surfaces are rendered in a descending order of expansion. Afterwards, ascending order and enabled back-face culling are used, resulting in correct depth sorting.

\section{Results}

The presented approach for generating hulls was tested for 1 proband and 5 patient datasets. For this purpose, major white matter tract systems were reconstructed in a first step, using streamline-based fiber tracking with a constant step size of $1 \mathrm{~mm}$ and user-defined ROIs. From the resulting fiber bundles, hulls were generated by means of rasterization, volume filtering, isosurface extraction and surface smoothing, as outlined in the previous sec- tions. The computing times for the hull surfaces shown in this paper were between $10 \mathrm{~s}$ and $3 \mathrm{~min}$ (on a PC equipped with a $\mathrm{P} 43.0 \mathrm{GHz}$ and $2 \mathrm{~GB}$ RAM), depending on the number of fibers which has to be rasterized and the number of iterations chosen for the surface filter. From various experiments, the following parameters proved to be adequate for generating hulls tightly fitting the underlying fiber tract, as shown in figure 7 :

- voxel size of rasterization volume: $1 \times 1 \times 1 \mathrm{~mm}^{3}$;

- size of gaussian filter kernel: $3 \times 3 \times 3$;

- isovalue for marching cubes: $0-10$;

- surface filter: scale-dependent Laplace filter;

- filter parameter $\lambda: 0.5$;

- number of filter iterations: 10-60.

One neurosurgeon and two medical students (neurosurgery) applied the approach to several tract systems in 

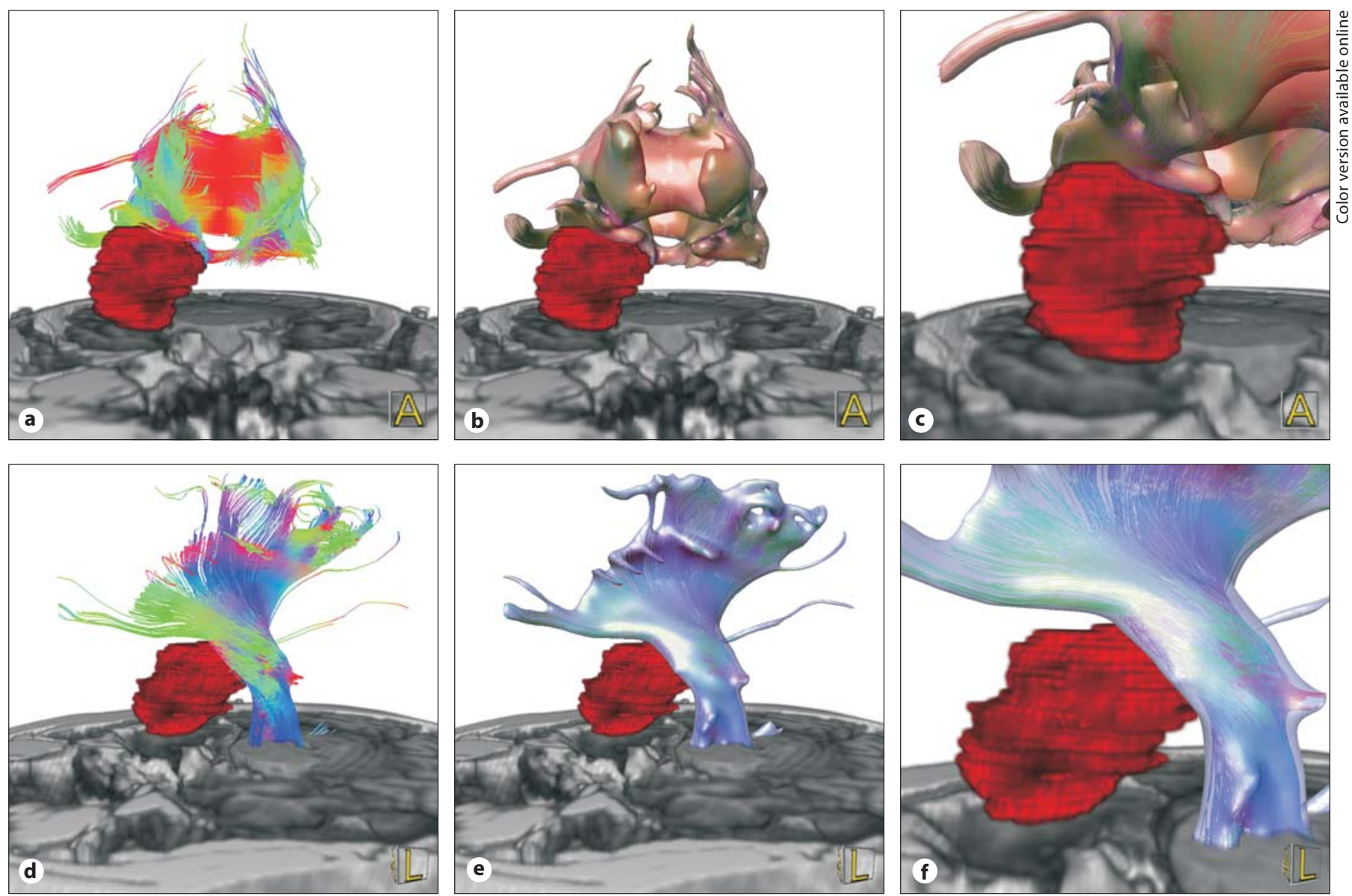

Fig. 9. Brain tumor patient. The corpus callosum (a-c) and the pyramidal tract (d-f) are very close to the tumor (dark gray; red in the online version), as indicated by fiber tracking (a, d). A hull wrapping the corpus callosum and the pyramidal tract was gener- ated (isovalue 1, scale-dependent Laplace filter with $\lambda=0.5,40$ iterations) which tightly fits the underlying fiber tracts $(\mathbf{b}, \mathbf{e})$. Zoomed views of the tumor region $(\mathbf{c}, \mathbf{f})$. different patients, namely the pyramidal tract, the optical tract and the corpus callosum. Manual segmentation of the required ROIs, fiber tracking and hull extraction with parameter adjustment required at most $10 \mathrm{~min}$ per white matter tract system. In figure 8 , the pyramidal tract in 4 patients is investigated, and the hulls as well as a semitransparent representation are shown. The quality of the hulls was visually confirmed by the medical experts based on semi-transparent hull visualization. In figure 9, hulls for the corpus callosum and the pyramidal tract are shown for the fifth brain tumor patient. The tumor was manually segmented and is shown in dark gray (red in the online version). Since the presented tract systems are very close to the lesion, intraoperative visualization of the respective hulls provides surgical guidance and supports safe resection. The quality of a hull resulting from a spe- cific parameter set can be visually investigated by simultaneous visualization of the fiber tract and the respective hull. In this way, the hull extraction parameters are easily adjusted in order to obtain tight hulls wrapping the underlying fiber tract.

In order to further support quality control and to provide a quantitative measure, it is also possible to measure the tightness of a hull with respect to the underlying tract system. For this purpose, the distance of each surface point to the nearest tract vertex is computed and evaluated (average and maximum value of all surface vertices). In order to efficiently compute the minimum distance for each surface vertex, the volume is decomposed into cubes with edge lengths of $4 \mathrm{~mm}$, and the tract vertices are assigned to the respective cube. For each surface vertex, the corresponding cube is determined and the minimum 
Table 1. In 5 patients, the pyramidal tract was reconstructed and wrapped with hulls (isovalue 5, scale-dependent Laplace filter with $\lambda=0.5)$

\begin{tabular}{rll}
\hline & \multicolumn{2}{l}{ Pyramidal tract } \\
\cline { 2 - 3 } & average, mm & maximum, mm \\
\hline 40 iterations & & \\
Patient 1 & 0.96 & 1.81 \\
Patient 2 & 1.09 & 1.97 \\
Patient 3 & 0.86 & 1.79 \\
Patient 4 & 0.92 & 1.89 \\
Patient 5 & 1.12 & 2.33 \\
50 iterations & & \\
Patient 1 & 0.89 & 1.75 \\
Patient 2 & 1.02 & 1.90 \\
Patient 3 & 0.80 & 1.74 \\
Patient 4 & 0.83 & 1.82 \\
Patient 5 & 1.06 & 2.05 \\
60 iterations & & \\
Patient 1 & 0.79 & 1.63 \\
Patient 2 & 0.96 & 1.85 \\
Patient 3 & 0.78 & 1.71 \\
Patient 4 & 0.75 & 1.77 \\
Patient 5 & 1.01 & 1.97 \\
\hline
\end{tabular}

The number of iterations for the surface filter was varied between 40, 50 and 60 iterations. For each hull, the average and maximum distance of all surface points to the nearest tract vertex was computed.

distance between the surface vertex and all tract vertices within the cube is computed. If the surface vertex is very close $(<1 \mathrm{~mm})$ to one of the sides of the cube, the tests are also performed for the tract vertices contained within the respective adjacent cube. As a result, the distance between a surface vertex with respect to the fiber tract is assessed. In table 1, the results for the pyramidal tract for the 5 patients are presented, depending on the number of iterations for the surface filter (the remaining parameter settings were according to the recommendation above, with an isovalue of 5). For each patient, hulls for the pyramidal tract were extracted after 40,50 and 60 surface filter iterations, and the average and maximum distance value for all surface points was determined.

Visual inspection of the hulls showed that the hulls enclosed the pyramidal tracts completely, only in case of 60 iterations, the tips of the tract systems stuck out a bit. The distance measurements further confirm the quality of the hulls, with an average distance between hull and tract of about $1 \mathrm{~mm}$. Moreover, these measurements are an upper bound of the real distance. Since the fiber tracking step size was set to $1 \mathrm{~mm}$, the real distance between surface vertex and fiber streamline may be up to $0.5 \mathrm{~mm}$ lower than the computed value. Nevertheless, the distance measures provide additional information about the tightness of the hulls and allow to quantitatively assess their quality.

\section{Discussion}

The primary objective of this work is to improve fiber tract visualization for medical application by providing smooth hulls tightly fitting the underlying fiber tracts. Since streamline representations only illustrate a direction, the shape of a streamline bundle as a whole can only be estimated. For this reason, hulls are necessary for intraoperative visualization. The presented approach makes it possible to display the boundary curves of fiber tracts in the focus plane of the OR microscope for neuronavigation. As a result, image-guided surgery based on hulls of important structures considerably reduces the risk of postoperative deficits, which is of high value for neurosurgery.

Since the fibers obtained from fiber tracking serve as a basis for hull generation, the quality of the hulls depends on the reliability of the fiber tracts. Even though the accuracy of streamlines is not the focus of this work, the potential risk of tracking errors resulting in fiber bundles which do not represent the whole neuronal structure should be noted. However, for the major tract systems considered in this work consisting of thick, coherent neuronal fibers, strong anisotropic diffusion is encountered. For this reason, fiber tracking algorithms are sufficiently well capable of recovering the underlying neuronal structures in these cases, as reported in a tracer experiment [29] and in clinical studies [7, 8, 30]. However, the applicability of fiber tracking and consequently the hull approach presented in this work may still be limited due to infiltration of the fibers by the tumor. In these cases, it is not possible to obtain sufficient clinical information from fiber tracking, and intraoperative cortical and subcortical mapping with or without awake surgery is necessary. According to preliminary clinical experiences, this is a general limitation of fiber tracking and the presented hull approach.

The usability and adjustment of hulls was tested by a neurosurgeon and two medical students (neurosurgery). They applied the approach to several tract systems in different patients, namely the pyramidal tract, the optical 
tract and the corpus callosum. They greatly appreciated the possibility to adjust the hulls according to their needs by varying mainly two parameters (the isovalue and the number of filter iterations), which provided enough flexibility for generating the desired hulls. After a short briefing, they soon developed an intuition for the parameter settings in order to generate the hulls they needed.

With respect to the quality of the obtained hulls, the presented processing pipeline meets the requirements for surgical planning. Adjustment of the hull extraction parameters in conjunction with visual inspection by medical experts allows to tightly fit the hull to the underlying fiber tract, especially in regions which are of interest for surgery. For this purpose, a combined visualization of the hull together with the underlying fiber tract is provided. For better comparison, visual inspection is further supported by a semi-transparent visualization of the hull. Additionally, the maximum and average distance of all surface points to the closest tract vertex is computed on demand for quality control, providing a quantitative measure of tightness. As a result, it is possible to further assess the quality of a hull beyond visual inspection.

According to the distance measurements, the hulls generated by the medical experts wrap the tract systems with an average distance of about $1 \mathrm{~mm}$ to the underlying fibers. With respect to accuracy, this slight average expansion is tolerable for surgery. In recent research, it was even suggested to adhere to a worst-case safety distance of about $5 \mathrm{~mm} \mathrm{[31]} \mathrm{in} \mathrm{order} \mathrm{to} \mathrm{compensate} \mathrm{for} \mathrm{effects} \mathrm{in-}$ fluencing the precision of location, such as brain shift and image distortions inherent to DTI data.

In comparison to other current approaches for wrapping fibers $[16,20]$, the presented technique has several advantages. Geometric wrapping [16] defines a centerline for the tract and defines boundary curves within planes perpendicular to the centerline, which are finally connected by a mesh. Consequently, this approach is restricted to elongated tracts, where no branching or diverging fiber directions occur. These situations are better captured based on tetrahedralization and 3-dimensional $\alpha$ shapes [20]. The resulting surface meshes provide high precision since the sampling points of the streamlines are reused. However, tetrahedralization requires dense bundles with no sparse regions in order to wrap all streamlines, and the resulting surfaces are not very smooth. In comparison, the presented technique is able to wrap fiber bundles of arbitrary shape and density. Even single streamlines diverging from the bundle and sparse regions are wrapped. Another approach potentially suitable for wrapping fiber bundles is the constrained elastic surface net [15]. Thereby, vertices are positioned within the border voxels of the rasterized volume and are connected by an initial mesh. In order to smooth the resulting surface mesh, relaxation iterations are performed with the constraint that a vertex remains within its host voxel. However, even after a high number of iterations, the hulls are not smooth. Furthermore, the iteration parameter is the only parameter for hull adjustment, restricting user interaction. To the contrary, the hulls resulting from the presented approach completely cover the underlying fiber tract and are easily adjusted based on several hull generation parameters. Robust and controlled surface generation and visual verification make the approach a powerful tool for medical application.

\section{Conclusion}

We presented a processing pipeline adapted to medical requirements for generating hulls encompassing neuronal pathways. The approach has different advantages over existing techniques and provides enough flexibility to wrap tract systems of arbitrary shape. The parameters for wrapping can be adjusted resulting in hulls tightly fitting the underlying fiber bundle. Overall, the presented techniques are of high value for medical application and intraoperative visualization in neurosurgery.

\section{Acknowledgments}

This work was supported by the Deutsche Forschungsgemeinschaft in the context of SFB 603, Project C9 and the Graduate Research Center '3D Image Analysis and Synthesis'.

\section{References}

Stereotact Funct Neurosurg 2009;87:50-60
1 Basser P, Pierpaoli C: Microstructural and physiological features of tissues elucidated by quantitative diffusion tensor MRI. J Magn Reson B 1996;111:209-219.

2 Basser P, Jones D: Diffusion-tensor MRI: theory, experimental design and data analysis - A technical review. NMR Biomed 2002; 15:456-467.

3 Enders F, Iserhardt-Bauer S, Hastreiter P, Nimsky C, Ertl T: Hardware-accelerated glyph-based visualization of major white matter tracts for analysis of brain tumors; in Galloway RL Jr, Cleary KR (eds): Visualization, Image-Guided Procedures, and Display. San Diego, SPIE, 2005, pp 504-511.

4 Kindlmann G: Superquadric tensor glyphs. Proc EG/IEEE TCVG VisSym, Konstanz, 2004, pp 147-154. 
5 Basser P, Pajevic S, Pierpaoli C, Duda J, Aldroubi A: In vivo fiber tractography using DT-MRI data. Magnet Reson Med 2000;44: 625-632.

6 Mori S, van Zijl P: Fiber tracking: principles and strategies - A technical review. NMR Biomed 2002;15:468-480.

7 Berman J, Berger M, Mukherjee P, Henry R: Diffusion-tensor imaging-guided tracking of fibers of the pyramidal tract combined with intraoperative cortical stimulation mapping in patients with gliomas. Neurosurgery 2004;101:66-72.

8 Nimsky C, Ganslandt O, Hastreiter P, Wang R, Benner T, Sorensen A, Fahlbusch R: Preoperative and intraoperative diffusion tensor imaging-based fiber tracking in glioma surgery. Neurosurgery 2005;56:130-138.

9 Behrens T, Johansen-Berg H, Woolrich M, Smith S, Wheeler-Kingshott C, Boulby P, Barker G, Sillery E, Sheehan K, Ciccarelli O, Thompson A, Brady J, Matthews P: Non-invasive mapping of connections between human thalamus and cortex using diffusion imaging. Nat Neurosci 2003;6:750-757.

10 Jackowski M, Kao C, Qiu M, Constable R, Staib L: Estimation of anatomical connectivity by anisotropic front propagation and diffusion tensor imaging; in Barillot C, Haynor DR, Hellier P (eds): Medical Image Computing and Computer-Assisted Intervention MICCAI 2004. Berlin, Springer, 2004, pp 663-670.

11 Merhof D, Richter M, Enders F, Hastreiter P, Ganslandt O, Buchfelder M, Nimsky C, Greiner G: Fast and accurate connectivity analysis between functional regions based on DT-MRI; in Larsen R, Nielsen M, Sporring J (eds): Medical Image Computing and Computer-Assisted Intervention - MICCAI 2006. New York, Springer, 2006, part II, pp 225-233.

12 Weinstein D, Kindlmann G, Lundberg E: Tensorlines: advection-diffusion based propagation through diffusion tensor fields. Proc IEEE Visualization, San Francisco, 1999, pp 249-253.
13 Lorensen W, Cline H: Marching cubes: a high resolution $3 \mathrm{D}$ surface reconstruction algorithm; in Stone MC (ed): Computer Graphics (Proceedings of SIGGRAPH '87). New York, ACM press, 1987, vol 21, pp $163-$ 169.

14 Nielson G: Dual marching cubes. Proc IEEE Visualization, Austin, 2004, pp 489-496.

15 Gibson SF: Constrained elastic surface nets: generating smooth surfaces from binary segmented data; in Wells WM, Colchester A, Delp S (eds): Medical Image Computing and Computer-Assisted Intervention - MICCAI 1998. Berlin, Springer, 1998, pp 888-898.

16 Enders F, Sauber N, Merhof D, Hastreiter P, Nimsky C, Stamminger M: Visualization of white matter tracts with wrapped streamlines. Proc IEEE Visualization, Minneapolis, 2005, pp 51-58.

17 Brun A, Knutsson H, Park H, Shenton M, Westin CF: Clustering fiber tracts using normalized cuts; in Barillot C, Haynor DR, Hellier P (eds): Medical Image Computing and Computer-Assisted Intervention - MICCAI 2004. Berlin, Springer, 2004, pp 368 375.

18 Maddah M, Mewes A, Haker S, Grimson E, Warfield S: Automated atlas-based clustering of white matter fiber tracts from DTMRI; in Duncan J (ed): Medical Image Computing and Computer-Assisted Intervention - MICCAI 2005. Berlin, Springer, 2005, pp 188 195.

19 Moberts B, Vilanova A, van Wijk J: Evaluation of fiber clustering methods for diffusion tensor imaging. Proc IEEE Visualization, Minneapolis, 2005, pp 65-72.

20 Merhof D, Meister M, Bingöl E, Hastreiter P, Nimsky C, Greiner G: Generation of hulls encompassing neuronal pathways based on tetrahedralization and $3 \mathrm{D}$ alpha shapes; in Horsch A, Deserno T, Handels H, Meinzer HP, Tolxdorff T (eds): Bildverarbeitung für die Medizin 2007. Berlin, Springer, 2007, pp 308-312.

21 Stalling D, Hege HC: Fast and resolution independent line integral convolution. Proc SIGGRAPH 1995, Los Angeles, 1995, pp 249-256.
22 Bresenham J: Algorithm for computer control of a digital plotter. IBM Syst J 1965;4: 25-30.

23 Kaufman A, Shimony E: 3D scan-conversion algorithms for voxel-based graphics. Proc ACM Workshop on Interactive 3D Graphics, Chapel Hill, 1986, pp 45-76.

24 Kobbelt L, Campagna S, Vorsatz J, Seidel HP: Interactive multi-resolution modeling on arbitrary meshes. Comput Graph 1998;32:105114.

25 Pinkall U, Polthier K: Computing discrete minimal surfaces and their conjugates. Exp Math 1993;2:15-36.

26 Bade R, Haase J, Preim B: Comparison of fundamental mesh smoothing algorithms for medical surface models. Proc Simulation Visualisierung, Magdeburg, 2006, pp 289304.

27 Desbrun M, Meyer M, Schröder P, Barr A: Implicit fairing of irregular meshes using diffusion and curvature flow. Proc SIGGRAPH, Los Angeles, 1999, pp 317-324.

28 Kim B, Rossignac J: GeoFilter: geometric selection of mesh filter parameters. Comput Graph Forum 2005;24:295-302.

29 Dauguet J, Peled S, Berezovskii V, Delzescaux T, Warfield S, Born R, Westin CF: 3D histological reconstruction of fiber tracts and direct comparison with diffusion tensor MRI tractography; in Larsen R, Nielsen M, Sporring J (eds): Medical Image Computing and Computer-Assisted Intervention - MICCAI 2006. New York, Springer, 2006, pp 109116.

30 Kamada K, Todo T, Masutani Y, Aoki S, Ino K, Takano T, Kirino T, Kawahara N, Morita A: Combined use of tractography-integrated functional neuronavigation and direct fiber stimulation. Neurosurgery 2005;102:664627.

31 Nimsky C, Ganslandt O, Fahlbusch R: Implementation of fiber tract navigation. Neurosurgery 2006;58:292-304. 